\title{
Magnetic Shielding Effectiveness Measurement of Magnetic Steel Sheets in ELF Range
}

\author{
Kyoheum Yeon ${ }^{1}$, Derac Son ${ }^{1 *}$, Eon Byeong Park², Jae Young Lee², Kyung Hwan Do ${ }^{3}$, and Jae Seg Park \\ ${ }^{1}$ Department of Physics, Hannam University, Daejeon 306-791, Korea \\ ${ }^{2}$ Environment Research Center, RIST Hyoja-dong 32 Pohang, Korea \\ ${ }^{3}$ Cold Rolled Product Technology Team, POSCO Center Daechi-dong 4 Seoul, Korea
}

(Received 28 October 2008, Received in final form 2 December 2008, Accepted 3 December 2008)

\begin{abstract}
In this study, a new kind of instrument for measuring the magnetic shielding effectiveness (MSE) was developed using a double yoke; one a magnetizing yoke and the other a sensing yoke. Using the developed instrument, the MSE could be measured for a steel sheet specimen in the ELF range, where the magnetic permeability contributes to the MSE at low frequencies and eddy currents contributes to the MSE high frequencies with $<0.1 \mathrm{~dB}$ reproducibility. The developed measuring method can be applied to quality control in a steel sheet company producing EMI/EMC shielding materials
\end{abstract}

Keywords : magnetic field shield, magnetic shielding effectiveness, EMI/EMC, double yoke, magnetic shielding factor

\section{Introduction}

The magnetic field shield from household appliances and power lines has become more important not only because of EMI/EMC problems but also because of the potential harmful effects on the human body [1,2]. Many countries now have regulations for an allowable magnetic field strength in the ELF range [3].

Methods for measuring the Magnetic Shielding Effectiveness (MSE) for constructions, such as cylinders or rectangular shapes, are given in the ASTM [4]. There are no standard methods available for measuring the MSE of a sheet specimen. However, it is necessary for a magnetic shielding material production company to know the magnetic shielding properties in a non-cylindrical shape, such as a sheet state, for quality control and to provide magnetic properties information from a sheet state

In this study, a new type of MSE measuring system was designed for a steel sheet specimen produced by POSCO.

\section{Principle Double Yoke Type MSE Measurement}

For a metallic magnetic material, two types of physical phenomena affect the magnetic field shield in the ELF

*Corresponding author: Tel: +82-42-629-7512

Fax: +82-42-629-8313, e-mail: deracson@hannam.ac.kr range. One is the high permeability of a magnetic material and the other is the skin effect due to the high conductivity and high permeability. A two yokes type of MSE measuring principle was used to determine, the above two effects in the same specimen, as shown in Fig. 1. Fig. 1(a) shows the magnetic shielding effect due to the high permeability. The top yoke is a magnetizing yoke and the bottom yoke is a sensing yoke. The test specimen was placed into the gap of the two yokes. When the specimen has high permeability, most of the magnetic flux lines move to the specimen and not to the sensing yoke. This decreases the induced voltage of the sensing coil. In the case of the skin effect, most of the magnetic flux lines do not penetrate into the specimen and the magnetic flux through the sensing yoke decreases. In addition, the voltage induced from the sensing coil decreases due to the magnetic shielding effect from the skin effect.

In this study, the magnetic shielding effectiveness $M S E$ was defined as the ratio of the induced voltage from the sensing coil, when the specimen is not put into between magnetizing and sensing yoke $V_{2}$ to the specimen is put into between magnetizing and sensing yoke $V_{1}$.

$$
M S E=V_{2} / V_{1}
$$

or

$$
\operatorname{MSE}(\mathrm{dB})=20 \log \left(V_{2} / V_{1}\right)
$$




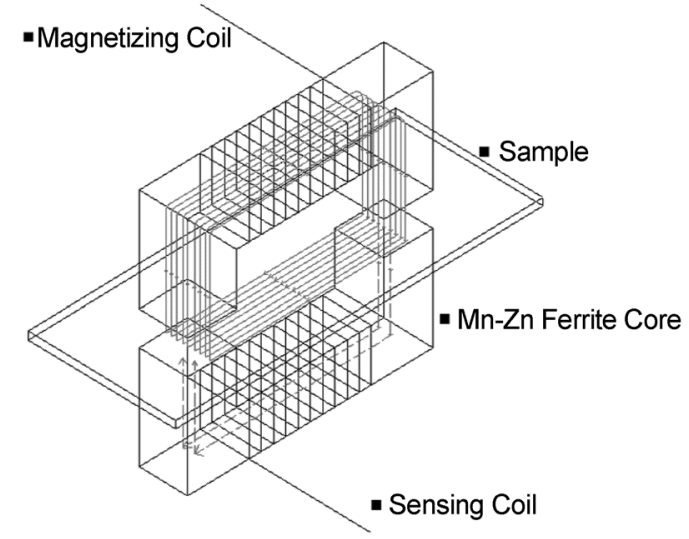

(a)

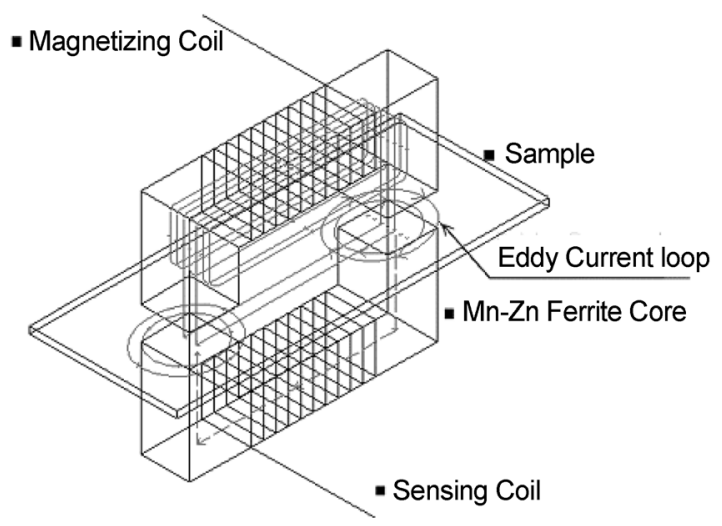

(b)

Fig. 1. Principle of double yoke type magnetic shielding effectiveness measurement; (a) in case of low frequency, (b) in case of high frequency.

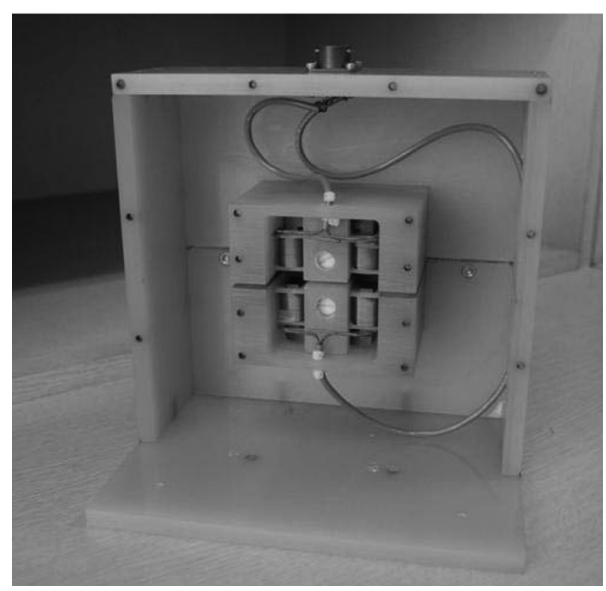

Fig. 2. Photograph of the constructed double yoke apparatus.

\section{Construction of MSE Measuring System}

Fig. 2 shows the constructed two-yoke type MSE measuring apparatus. A MnZn ferrite core was used as a yoke material and 4 coils with 120 turns were wound on the yokes. The pole face of the yoke was $7.7 \mathrm{~mm} \times 14.5 \mathrm{~mm}$, and distance between the centers of the yokes was 42.3 $\mathrm{mm}$. The gaps between the magnetizing yoke and speci-

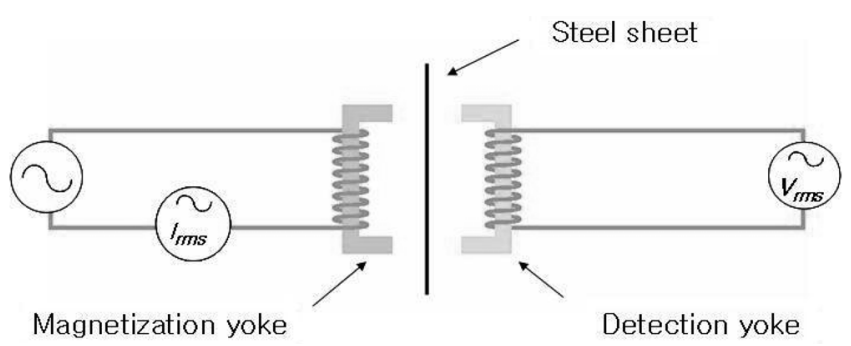

Fig. 3. Schematic diagram of the measuring system. men, and the magnetizing yoke and sensing yoke were fixed to $3 \mathrm{~mm}$ and $8 \mathrm{~mm}$ respectively. Fig. 3 shows a block diagram of the measuring system. The magnetizing voltage and frequency were generated using an oscillator and the magnetizing current $I_{\text {rms }}$ was measured using a digital voltmeter and precision shunt resistor. The voltage induced from the sensing yoke $V_{\text {rms }}$ was measured using a digital voltmeter.

\section{Experimental Results}

Non-oriented silicon steel $(0.5 \mathrm{~mm}$ thick) specimens with sizes ranging from $60 \mathrm{~mm} \times 60 \mathrm{~mm}$ to $200 \mathrm{~mm} \times$ $200 \mathrm{~mm}$ in $20 \mathrm{~mm}$ steps were prepared to observe specimen size affect to the MSE measurement. Fig. 4 shows the MSE as a function of frequency in the different speci-

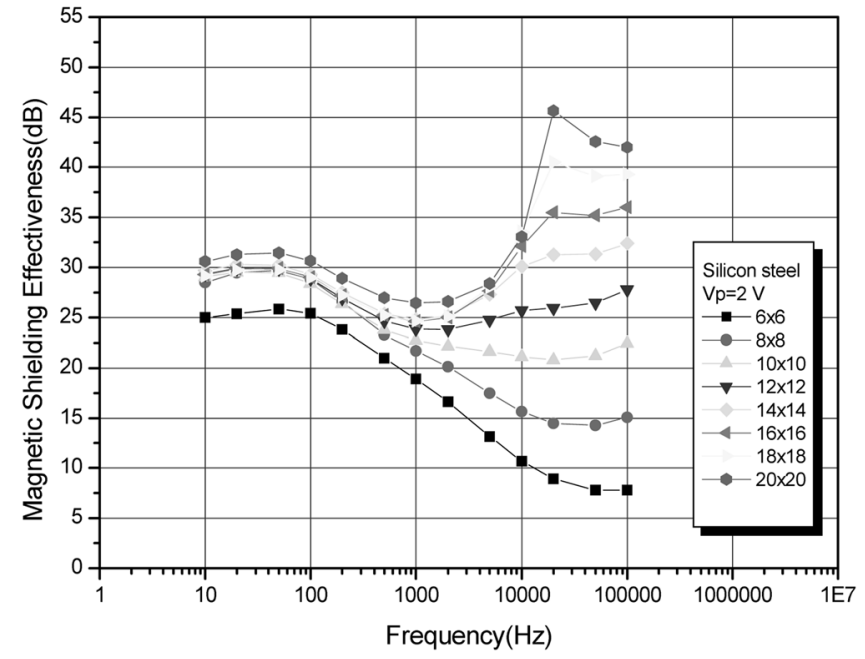

Fig. 4. Magnetic shielding effectiveness as a function of the frequency under different specimen sizes. 


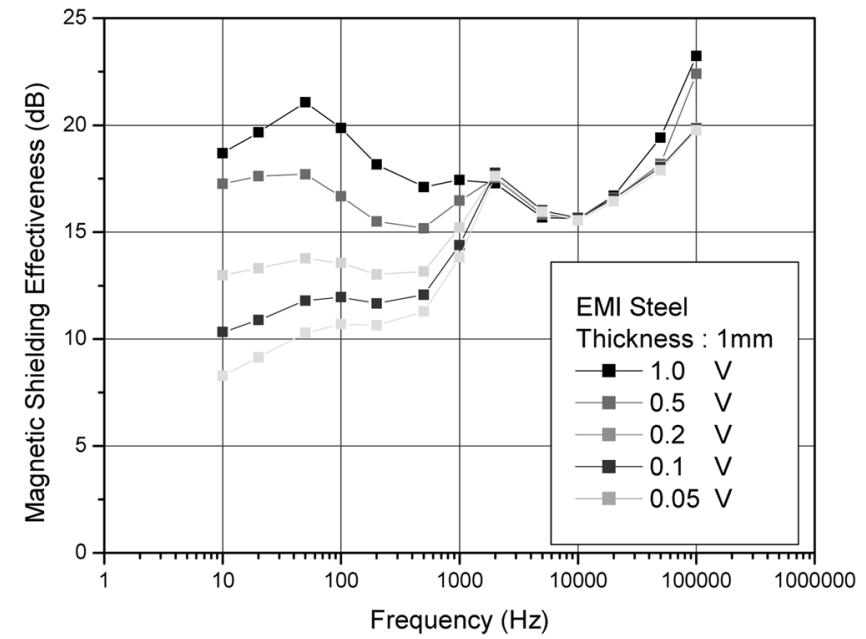

Fig. 5. Magnetic shielding effectiveness as a function of the frequency under different applied $\mathrm{AC}$ voltage for specimen size of $80 \mathrm{~mm} \times 80 \mathrm{~mm}$.

mens. At frequencies $<1 \mathrm{kHz}$, the magnetic permeability was the dominant contribution to MSE, but at frequencies

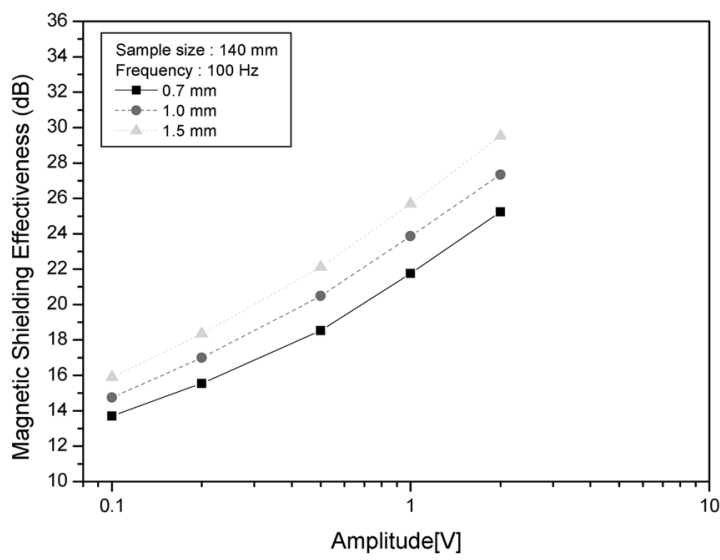

(a)

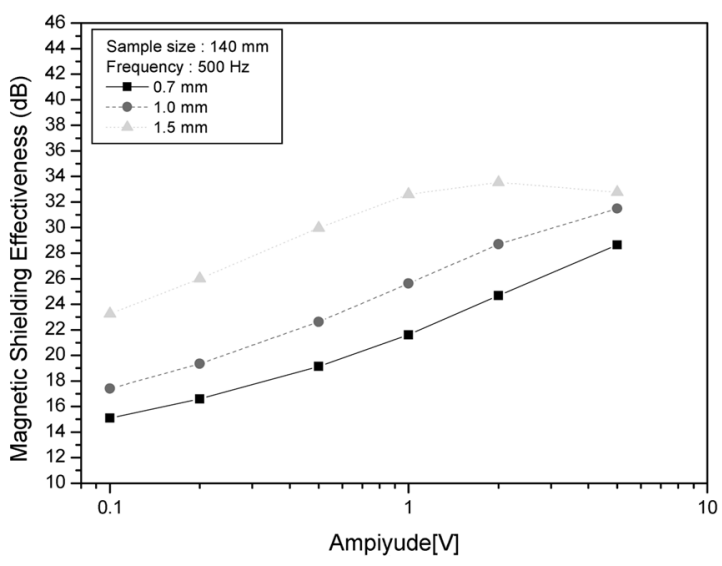

(c)
$>1 \mathrm{kHz}$, skin effects from eddy currents were dominant contribution to the MSE. Moreover, the MSE was strongly dependent on the specimen size. This means that the MSE originating from eddy current cannot increase when the specimen size is insufficient for eddy current loops. On the other hand, a suitable specimen size that does not distort the eddy current loop is needed for higher precision and reproducibility. Fig. 5 shows the MSE of the POCSO developed EMI steel sheet as a function of frequency under different applied voltages. In the eddy current region, the MSE was relatively unaffected by applied AC voltage. However, in the permeability contributing region, the MSE changed considerably with the applied AC voltage because the permeability of the specimen changed with the applied AC voltage. Fig. 6 shows the MSE as a function of the applied AC voltage of $1 \mathrm{kHz}$ under different specimen thicknesses for a specimen size of $140 \mathrm{~mm}$ $\times 140 \mathrm{~mm}$. In the case of a $1.5 \mathrm{~mm}$ thickness and a frequency of $500 \mathrm{~Hz}$, the MSE becomes saturated above an applied AC voltage of $1 \mathrm{~V}$. This means that $1 \mathrm{~V}$ is the maximum applicable voltage for a frequency of $500 \mathrm{~Hz}$

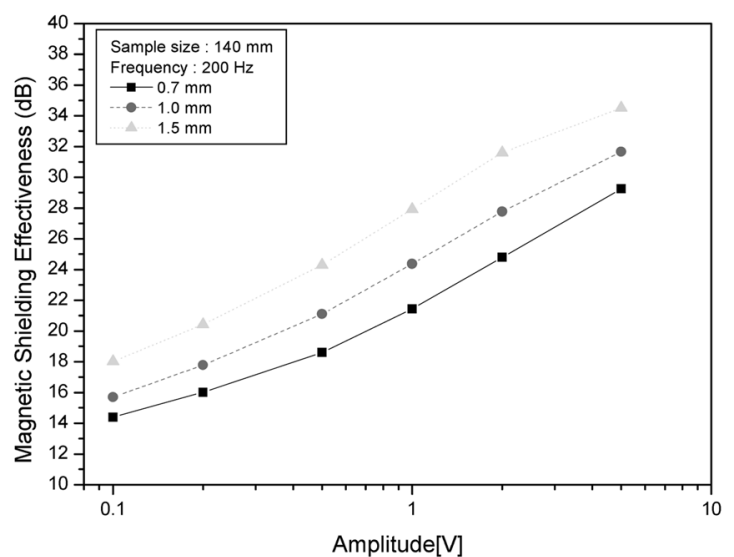

(b)

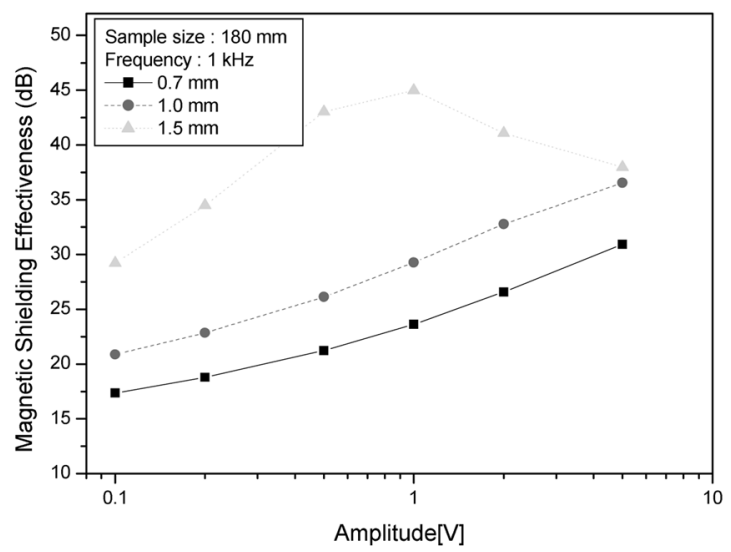

(d)

Fig. 6. Magnetic shielding effectiveness as a function of the applied AC voltage for different frequency and thickness of specimens. 


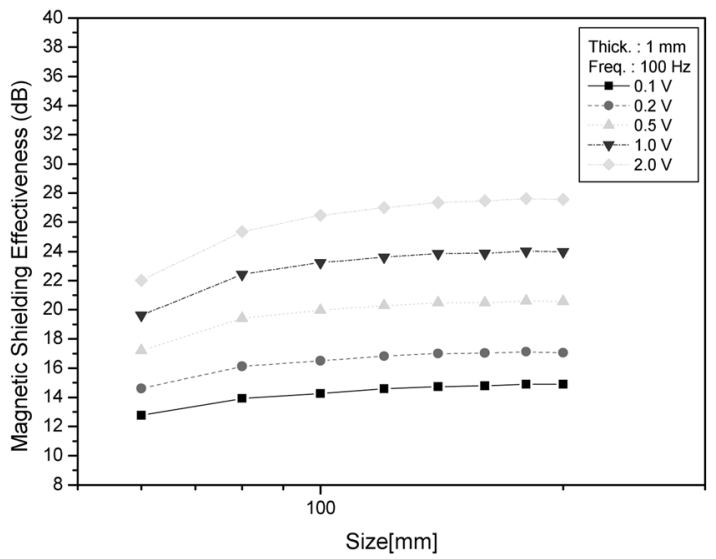

(a)

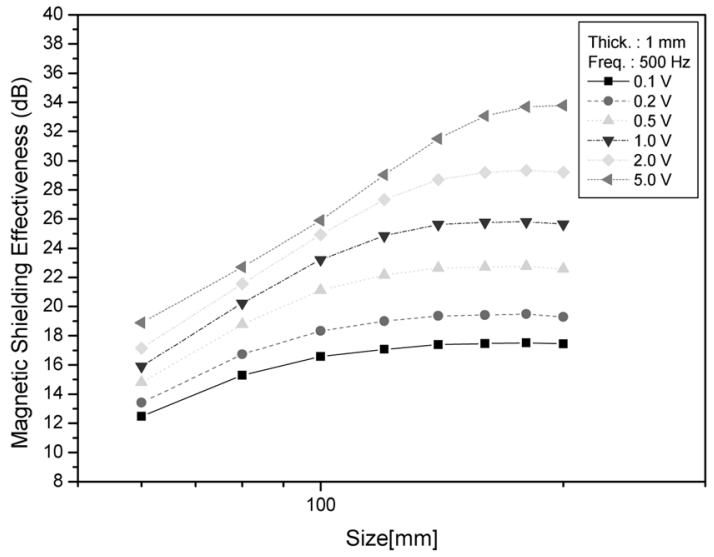

(c)

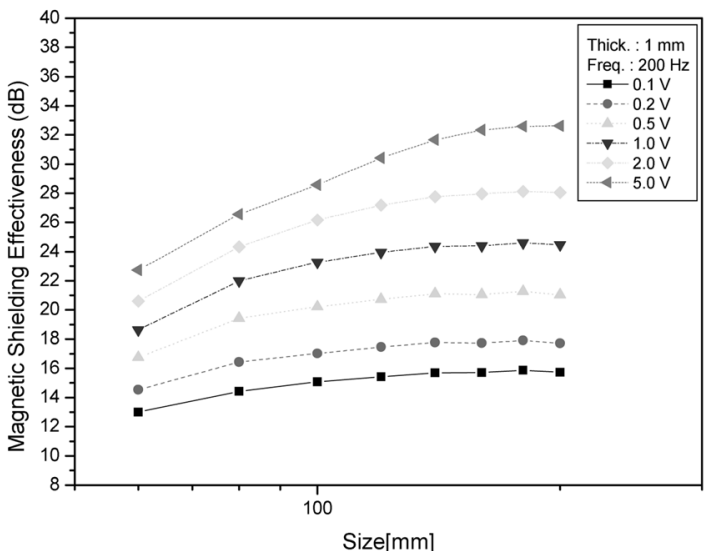

(b)

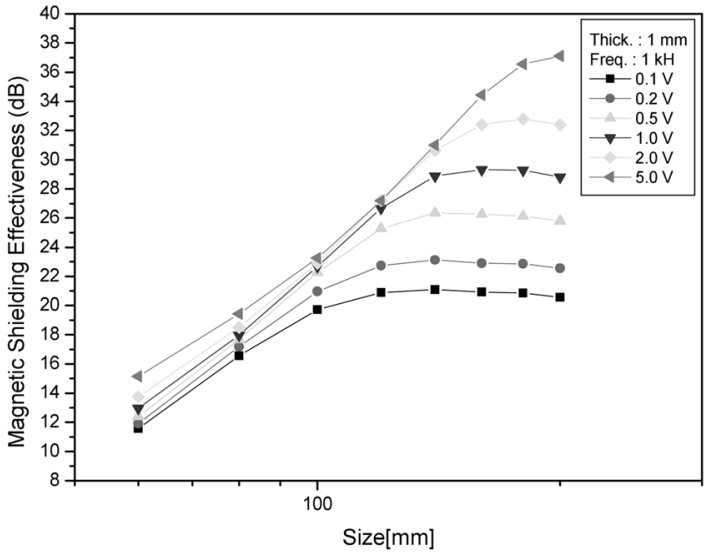

(d)

Fig. 7. Magnetic shielding effectiveness as a function of the specimen size for different applied AC voltages and frequencies.

and a specimen thickness of $1.5 \mathrm{~mm}$. Fig. 7 shows the MSE as a function of the specimen size for the different frequencies and applied AC voltages. For a given voltage and specimen thickness, a larger specimen size is needed when the frequency increases. The MSE measurement up to $1 \mathrm{kHz}$ and $1 \mathrm{~V} \mathrm{AC}$ for a $1.5 \mathrm{~mm}$ thick specimen using the developed double yoke method required a $140 \mathrm{~mm} \times$ $140 \mathrm{~mm}$ sized specimen. The reproducibility of the MSE measurement using the developed measuring system was better than $0.1 \mathrm{~dB}$.

\section{Conclusions}

In this stduy, a double yoke type instrument was developed for measuring the magnetic shielding effectiveness (MSE). Using the developed instrument, the MSE of a steel sheet specimen could be measured in the ELF range.
The results showed that the magnetic permeability contributes to the MSE at low frequencies and the eddy current contributes to the MSE at high frequencies with better than $0.1 \mathrm{~dB}$ reproducibility. The developed measuring method can be applied to the quality control in steel sheet companies producing EMI/EMC shielding materials.

\section{References}

[1] J. L. Norman Violette, D. R. J. White, and M. F Violette, Electromagnetic Compatibility Handbook, Van Nostrand Reinhold Co. 1987.

[2] K. Fitzgerald, "Electromagnetic fields: the jury's still out", IEEE spectrum Agust, pp. 22-35 (1990).

[3] www.inchem.org/documents/ehc/ehc35.htm.

[4] ASTM A698M-92. 